\title{
All-optical density downramp injection in electron-driven plasma wakefield accelerators
}

\author{
D. Ullmann $\odot,{ }^{1,2,3, *}$ P. Scherkl $\odot,{ }^{1,2,4, *}$ A. Knetsch $\odot,{ }^{5}$ T. Heinemann $\odot,{ }^{1,2,6,7}$ A. Sutherland, ${ }^{1,2,8}$ A. F. Habib $\odot,{ }^{1,2}$ \\ O. S. Karger, ${ }^{7}$ A. Beaton ${ }^{1},{ }^{1,2}$ G. G. Manahan, ${ }^{1,2}$ A. Deng, ${ }^{9}$ G. Andonian, $,{ }^{9}, 10$ M. D. Litos, ${ }^{11}$ B. D. O’Shea ${ }^{\circ},{ }^{8}$ \\ J. R. Cary $\odot,{ }^{11,12}$ M. J. Hogan $\odot{ }^{8}{ }^{8}$ V. Yakimenko, ${ }^{8}$ J. B. Rosenzweig, ${ }^{9}$ and B. Hidding ${ }^{1,2}$ \\ ${ }^{1}$ Department of Physics, University of Strathclyde, SUPA, Glasgow G1 1XQ, United Kingdom \\ ${ }^{2}$ Cockcroft Institute, Daresbury, Warrington WA4 4AD, United Kingdom \\ ${ }^{3}$ Faculty of Physics and Astronomy, Friedrich-Schiller-Universität Jena, 07743 Jena, Germany \\ ${ }^{4}$ University Medical Center Hamburg-Eppendorf, University of Hamburg, 20246 Hamburg, Germany \\ ${ }^{5}$ LOA, ENSTA Paris, CNRS, Ecole Polytechnique, Institut Polytechnique de Paris, 91762 Palaiseau, France \\ ${ }^{6}$ Deutsches Elektronen-Synchrotron, 22607 Hamburg, Germany \\ ${ }^{7}$ Department of Experimental Physics, University of Hamburg, 20148 Hamburg, Germany \\ ${ }^{8}$ SLAC National Accelerator Laboratory, Menlo Park, California 94025, USA \\ ${ }^{9}$ Department of Physics and Astronomy, University of California Los Angeles, Los Angeles, California 90095-1547, USA \\ ${ }^{10}$ Radiabeam Technologies, Santa Monica, California 90404, USA \\ ${ }^{11}$ Center for Integrated Plasma Studies, Department of Physics, University of Colorado, Boulder, Colorado 80309-0390, USA \\ ${ }^{12}$ Tech-X Corporation, Boulder, Colorado 80303, USA
}

(Received 18 July 2020; revised 15 August 2021; accepted 9 November 2021; published 8 December 2021)

\begin{abstract}
The properties of electron beams obtainable from plasma wakefield accelerators are largely determined by their injection into the plasma wave. Density downramps can facilitate injection of plasma electrons and are realized routinely in laser wakefield accelerators by gas-dynamic production of longitudinal downramp profiles. We show that density downramp injection is in fact a multidimensional process when operated below the spatiotemporal scales of the plasma wave. In beam-driven plasma waves, unlocking this interaction regime by microscopic plasma torches allows the generation of unique electron beams, such as planar counteroscillating twin beamlets. Our findings generalize density downramp injection and extend its capabilities, which may find applications ranging from radiation production to high-energy-physics research.
\end{abstract}

DOI: 10.1103/PhysRevResearch.3.043163

\section{INTRODUCTION}

In electron-beam-driven [1-6] and laser-pulse-driven [7-12] plasma wakefield accelerators, transient charge separation of plasma electrons and ions creates plasma waves in the wake of the driver pulse. The properties of these plasma waves primarily depend on the plasma density $n_{\mathrm{e}}$, which determines the plasma frequency $\omega_{\mathrm{p}} \propto n_{\mathrm{e}}^{1 / 2}$ and wavelength $\lambda_{\mathrm{p}} \propto n_{\mathrm{e}}^{-1 / 2}$. They define the relevant spatiotemporal scales of the wave structure, namely, $1 / \omega_{p}$ of the order of a few tens to hundreds of femtoseconds and $\lambda_{p}$ of the order of a few tens to hundreds of microns for plasma densities in the range of $n_{e} \approx$ $10^{23}-10^{24} \mathrm{~m}^{-3}$. Such plasma waves provide ultrastrong accelerating and focusing electric fields, which scale as $E \propto n_{e}^{1 / 2}$ according to classical wave breaking $[13,14]$, and can reach tens to hundreds of $\mathrm{GV} / \mathrm{m}$ amplitudes. Plasma wakefield accelerators therefore offer an alternative to the unsustainably growing footprint of conventional particle accelerators [15]

\footnotetext{
*These authors contributed equally to this work.

Published by the American Physical Society under the terms of the Creative Commons Attribution 4.0 International license. Further distribution of this work must maintain attribution to the author(s) and the published article's title, journal citation, and DOI.
}

that are restricted to orders of magnitude weaker fields that are sustainable by much larger metallic cavities [16].

In addition to their functional role as accelerators, plasma wakefields also offer unique opportunities for designing integrated electron injectors: Electron populations originating from the plasma can be injected into the plasma wave, thus forming beams with femtosecond-scale durations and high currents. This obviates the need for additional beam compressors as required in conventional accelerators and the associated significant challenge of emittance and quality degradation due to coherent synchrotron radiation. At the same time, the rapid acceleration in the plasma wave limits space-charge-based emittance growth of injected electrons and allows for generating high-quality beams.

Various plasma injector types have been conceptualized [17-24] and realized [12,25-29]. Of these, density downramp injectors $[17,18,25,27,30-39]$ achieve injection by local modification of the accelerating plasma cavity and rely on negative longitudinal plasma density gradients $\partial_{x} n_{\mathrm{e}}(x)$ in the propagation direction of the plasma wave. This enlarges the plasma blowout structure by increasing the local plasma wavelength and reduces the wake phase velocity $v_{\text {ph }}(\xi)$ at a position $\xi=x-c t$ behind the driver [22], where $c$ is the speed of light. During this process, affected plasma electrons with suitably altered trajectories can be captured by the plasma wave to form the injected electron beam. 
Density downramp injection was originally proposed for laser-driven wakefield accelerators (LWFAs) in the form of gentle longitudinal downramps [17], i.e., longer than $\lambda_{\mathrm{p}}$, and for particle beam-driven plasma wakefield accelerators (PWFAs) in the form of steeper longitudinal downramps [18] shorter than $\lambda_{\mathrm{p}}$. Various schemes have been developed and implemented in LWFA experiments via modified gas targets. Creating density ramps by such means requires perturbing the gas medium such that it locally deviates from its natural tendency towards density equilibrium. Feasible techniques include tailored gas flows $[27,32,33]$, dynamic shocks in gas jets introduced by hardware obstacles [34-39], and laser-based heating and subsequent plasma expansion $[25,30,31]$.

In these schemes, the gas or ion density profile is manipulated on spatiotemporal scales much larger than those of the plasma wave. These gas-dynamic methods have been continuously developed and today constitute an experimentally viable standard injection strategy for LWFAs to produce high-quality electron beams.

Despite numerous numerical and theoretical studies [40-44], no successful experimental realization of gas-dynamically produced density downramp injection has been achieved in linear-accelerator-driven PWFA systems to date. This is remarkable, since the potential of density downramp injection $[18,45]$ as a source of high-brightness electron beams [46] was first discovered and proposed in the context of PWFAs. Gas density downramp-based injection remains a major goal of PWFA-capable facilities [41,47].

However, the PWFA does not inevitably rely on manipulation of the underlying gas density profile to realize plasma density profiles indirectly. Instead, plasma density profiles can be produced directly and even in homogeneous gas densities: While the multi-GV/m-scale electric fields of the particle driver efficiently can excite plasma waves in lowionization-threshold media, they leave the higher ionization levels of gas components intact. This opens the possibility to employ comparatively low-power laser pulses with intensities $I \approx 10^{14}-10^{16} \mathrm{~W} / \mathrm{cm}^{2}$ to selectively liberate electrons from higher ionization levels via tunneling ionization. The negligible ponderomotive force of laser pulses at such intensities produces localized, cold plasma density spikes or "torches," featuring plasma density downramps capable of injection $[48,49]$. In contrast to gas-dynamic techniques, the plasma torches are superimposed on the plasma employed for wake excitation and are thereby decoupled from the homogeneous and unaltered background plasma.

In the following, we report on the innovative capabilities that distinguish all-optical plasma torches from existing beam injectors. These arise from the versatility of optical focusing, which allows for injector density distributions beyond the limits of conventional density downramp techniques. Based on this perspective of downramp injection physics, we present a quantitative model predicting the injected charge as a result of the overlap of plasma wakefields with the torch density distributions, covering conventional and different effects (Sec. II). We then apply this formalism to experimental results from the first demonstration of the plasma torch process at the Facility for Advanced Accelerator Experimental Tests (FACET) at the SLAC National Accelerator Laboratory (Sec. III). These data exhibit first evidence of the microscopic dynamics

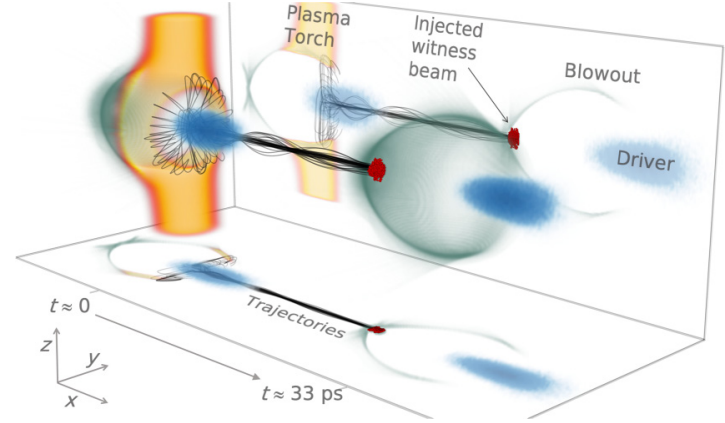

FIG. 1. Plasma torch injection based on 3D particle-in-cell simulations. The electron drive beam (blue) excites a plasma wave in the blowout regime (green). When the plasma wave traverses the plasma torch (orange), the locally increased density warps the plasma wave, which leads to injection of plasma electrons. Those subsequently form the witness beam (red). Selected trajectories of trapped electrons are displayed in the laboratory frame (black lines). See also [50] for the corresponding video.

arising from the similar spatiotemporal scales of the plasma wave and plasma torch. We conclude with an exploration of the further potential of multidimensional (three-dimensionally spatial and temporal] control of all-optical plasma density downramp injection and present alternative electron-beam configurations (Sec. IV) enabled by this approach.

\section{PLASMA TORCH INJECTION MODELING}

The plasma torch injection scheme is visualized in Fig. 1 based on a three-dimensional (3D) particle-in-cell (PIC) simulation using VSIM/VORPAL [51]. The electron drive beam propagates through a plasma with density $n_{\mathrm{ch}}$, generated from a low-ionization-threshold gas such as hydrogen, thereby exciting an intense trailing plasma wave in the blowout regime [2]. The plasma torch laser pulse (not shown) has already produced the perpendicular plasma torch density spike $n_{\mathrm{T}}$ from higher-ionization-threshold media such as helium, superimposing the hydrogen plasma. On the density downramp at the end of this torch, plasma electrons are captured by the plasma wave and form the injected and accelerated witness bunch.

Although the plasma torch displays typical characteristics of density downramp injectors, the density distribution generated by optical ionization clearly deviates from the established picture of purely longitudinal gradients. We therefore model the injection by means of the volumetric and temporal plasma density distribution $n_{\mathrm{T}}(\xi, y, z, t)$ together with the 3D distribution of the longitudinal wakefield component $E_{\xi}(\xi, y, z)$. We find (see Appendix A) that their overlap, expressed by the cross correlation operator $*$ acting in the longitudinal direction, can estimate the injected charge $Q_{\mathrm{M}}$ produced by density downramp injectors in general,

$$
Q_{\mathrm{M}} \approx-\frac{\epsilon_{0} C}{\lambda_{\mathrm{p}}}\left[\iint E_{\xi} *\left(\frac{\Delta \lambda_{\mathrm{p}}}{\lambda_{\mathrm{p}}}\right)^{3} d y d z\right]_{\min \xi},
$$

where the multidimensional function $\Delta \lambda_{\mathrm{p}} / \lambda_{\mathrm{p}}(\xi, y, z, t)=$ $1-\left(1+n_{\mathrm{T}} / n_{\mathrm{ch}}\right)^{-1 / 2}$ denotes the variation of the plasma wavelength induced by the density transition in terms of the 
background plasma density $n_{\text {ch }}$. The constant scaling factor $C=565$ ensures agreement of the model with PIC simulations and experimental data for all data presented in this work.

The cross correlation resembles the core of Eq. 1 and includes the density transition as well as the 3D-wakefield distribution enclosed by the blowout sheath. This macroscopic profile weights the accelerating field distribution and restricts the spatial integration to the intersection region. The minimum of the integrated cross correlation then is proportional to the injected charge. Descriptively, this minimum is reached approximately when the density downramp overlaps with the peak accelerating wakefield in the rear of the blowout. Equation (1) introduces volumetric and temporal dependences into density downramp physics modeling. Such volumetric effects include scenarios which trigger injection only in specific parts of the blowout, e.g., for narrow or spatially shifted torches, and temporal effects include plasma torches that dynamically evolve during the interaction process. Equation 1 yields absolute injected charge values directly from the overlapping wakefield and torch distributions. The model thus extends substantially beyond the conventional 1D interpretation of downramp physics and single-particle descriptions such as in [44] or scalings derived from experimental data [52].

To demonstrate the applicability of this model, we compare the predicted injected charge with results from 3D PIC simulations of the plasma torch injector. In this case, the density distribution $n_{\mathrm{T}}$ is determined by tunneling ionization. At positions where complete ionization is reached, excess laser intensity does not generate additional plasma, which gives rise to a typical flat-top density structure. For simplicity, we assume Gaussian focusing and approximate the resulting central plasma torch by a cylinder with radius $r_{\text {core }}$ of constant density. The superlinear dependence of ionization rates on the laser field typically yields steep density gradients surrounding the central region of full ionization, which we model as $\cos ^{2}$ shaped ramps of length $l_{\text {ramp }}$, in total expressed by

$$
\frac{n_{\mathrm{T}}}{n_{\mathrm{T}, 0}}(r, z)= \begin{cases}1, & r<r_{\text {core }} \\ \cos ^{2}\left(\frac{\pi}{2} \frac{r-r_{\text {core }}}{l_{\text {ramp }}}\right), & r_{\text {core }} \leqslant r<r_{\text {core }}+l_{\text {ramp }} \\ 0, & r>r_{\text {core }}+l_{\text {ramp }},\end{cases}
$$

where $z$ denotes the propagation direction of the plasma torch laser. These shapes are in good agreement with tunneling ionization calculations following [53] and with PIC simulations.

To compare injection yields from the model and PIC simulation, we explore two different regimes, namely, one with central torch diameter wider than the blowout [Fig. 2(a)] and one where the torch is thinner than the blowout [Fig. 2(b)]. In both regimes, we scan across steep to gentle ramp lengths and plot the injected charge in Figs. 2(c) and 2(d). For wide torch configurations, we find that the injected charge is maximal for shortest ramps and decreases monotonically towards longer ramps. This is known from classical gas density downramp injectors $[46,52,54]$ and shows that wide plasma torches can resemble their 1D gas-dynamic counterparts. In contrast, narrow torch distributions with core diameter much smaller than the blowout radius display a rapid decrease of injected charge once the ramp becomes shorter than $\sim \lambda_{p}$. In this case, the effective plasma torch extent shrinks and, notably, becomes
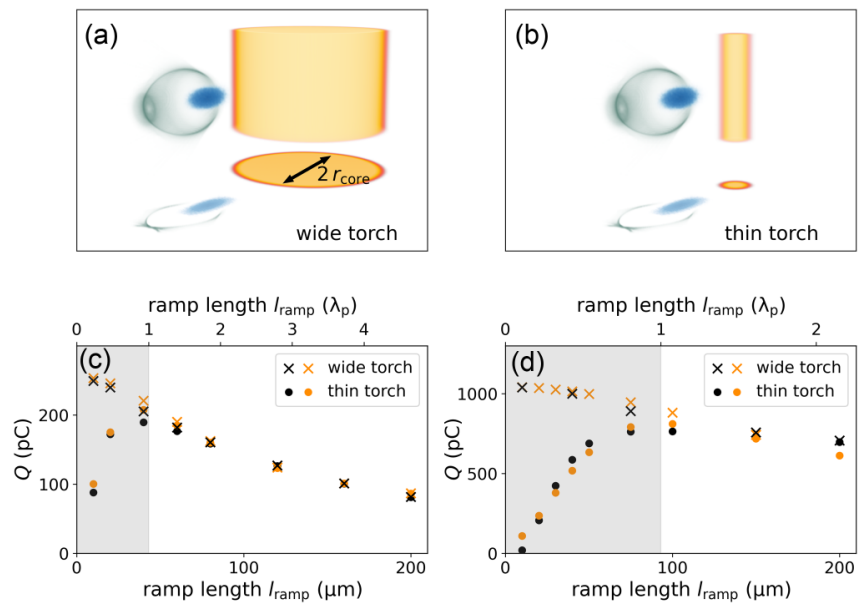

FIG. 2. Established and novel regimes of density downramp injection using plasma torches with $n_{\mathrm{T}, 0}=0.5 \times n_{\mathrm{ch}}$. Threedimensional PIC simulations use plasma torches with cores (a) wider and (b) narrower than the blowout. Varying the torch ramp length changes the injected charge $Q$ from the PIC simulation (black) and the model (orange) for PWFAs with plasma wavelengths (c) $\lambda_{\mathrm{p}} \approx 43 \mu \mathrm{m}$ and (d) $\lambda_{\mathrm{p}} \approx 93 \mu \mathrm{m}$ (d). The other parameters are (c) $r_{\text {core, wide }}=40 \mu \mathrm{m}$ and $r_{\text {core,thin }}=2.5 \mu \mathrm{m}$ and (d) $r_{\text {core, wide }}=$ $80 \mu \mathrm{m}$ and $r_{\text {core,thin }}=3.5 \mu \mathrm{m}$. Gray shades indicate regions $l_{\text {ramp }} \leqslant$ $\lambda_{\mathrm{p}}$. For narrow torches, these regions reveal a novel regime of density downramp injection unlocked by volumetric effects.

smaller than the blowout radius in the transverse direction. This reduces the volumetric overlap with the wakefield and yields less charge according to Eq. 1 .

Predictions by the model and values obtained from PIC simulations are in good agreement for the two different PWFA densities over the full range of scanned ramp lengths. These results emphasize that density downramp injection indeed represents a multidimensional process that has only been employed in its 1D limit so far. The all-optical plasma torch injector can realize and exploit these multidimensional aspects of density downramps.

\section{PLASMA TORCH DOWNRAMP INJECTION AT SLAC FACET}

We have realized an experimental demonstration of density downramp injection at FACET. In [29] we exploited the approach as an enabling diagnostic step to enter the plasma photocathode regime $[24,55]$. Here we demonstrate, by combining the present model, simulations, and experimental data, that the plasma torch technique enables volumetric and temporal density downramp injection control.

\section{A. Experimental setup}

We developed the capabilities required to explore and demonstrate plasma torch density downramp injection at SLAC FACET within the E-210 collaboration. The linear accelerator provided electron drive beams with charge $Q_{\mathrm{D}} \approx 3.2$ $\mathrm{nC}$, energy $W_{\mathrm{D}} \approx 20 \mathrm{GeV}$, length $\sigma_{\mathrm{rms}, x} \approx 25-40 \mu \mathrm{m}$, and typical widths at focus of $\sigma_{\mathrm{rms}, y} \approx 15-30 \mu \mathrm{m}$ and $\sigma_{\mathrm{rms}, z} \approx$ $20-30 \mu \mathrm{m}$. This drive beam was focused into an experimental 

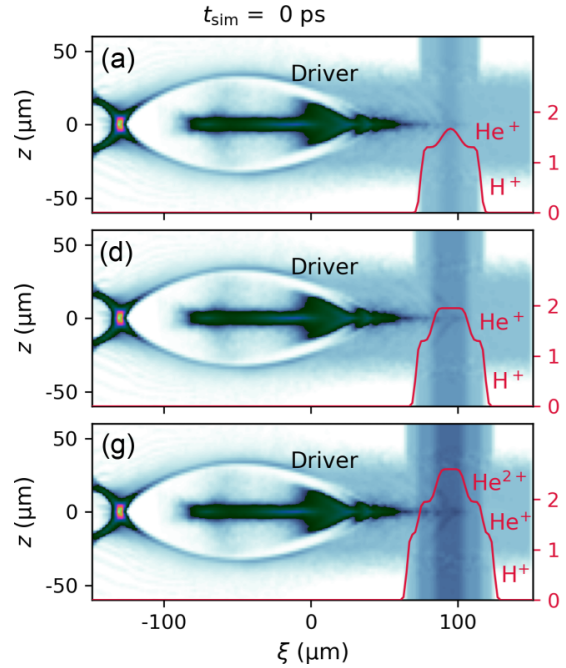
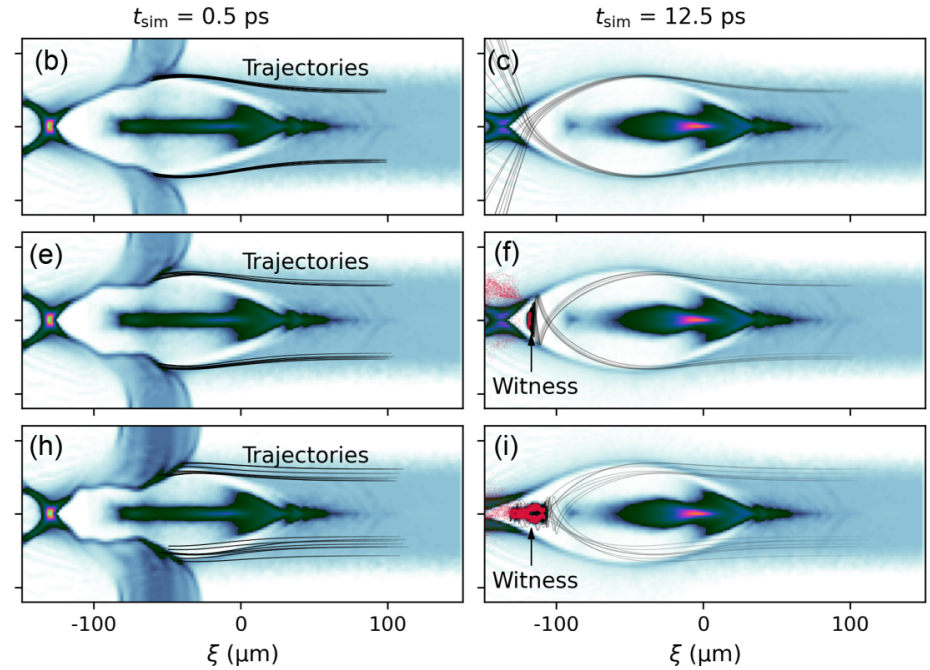

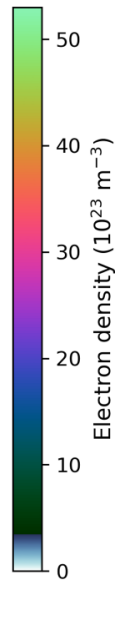

FIG. 3. Replication of the FACET experiment in 3D PIC simulations. Interactions with different torch distributions $n_{\mathrm{T}}$ from (a)-(c) 0.5 , (d)-(f) 1 , and (g)-(i) $5 \mathrm{~mJ}$ torch laser energy are shown at different times (a), (d), and (g) $t_{\text {sim }}=0$; (b), (e), and (h) $t_{\text {sim }}=0.5$; and (c), (f), and (i) $t_{\text {sim }}=12.5$ ps. The torch profiles (red) differ in composition, peak density, and volume as illustrated in (a), (d), and (g). The plasma torch modulates the blowout structure [(b), (e), and (h)] and selected trajectories show electron dynamics, which cause injection and witness beam formation for the cases in (f) and (i).

chamber filled with a premixed 50:50 hydrogen-helium gas mixture. Selective ionization of the hydrogen component was achieved by a laser pulse focused with a holographic axilens [56,57] that produced a plasma channel of $\sim 1 \mathrm{~m}$ length and maximum diameter of $\sim 100 \mu \mathrm{m}$ [29] to sustain the PWFA. The plasma channel density was $n_{\mathrm{ch}} \approx 1.3 \times$ $10^{23} \mathrm{~m}^{-3}$.

A separate laser arm was split off from the main laser and compressed to a FWHM duration of $\tau_{\mathrm{L}} \approx 64 \mathrm{fs}$. A fraction of this laser pulse was used for electro-optical sampling (EOS) upstream of the interaction area and another fraction was focused perpendicularly to the electron-beam path with an off-axis parabola $(f / 22.9)$ to a spot size of $w_{0, \mathrm{~L}} \approx 20 \mu \mathrm{m} \mathrm{rms}$ to generate the plasma torch at the interaction point. This laser pulse could be switched on and off, tuned in energy, and its spatial alignment and synchronization with the electron driver beam could be varied. This allowed for controlled generation of cold plasma torches with spatially and temporally modified density distributions $n_{\mathrm{T}}$ via local ionization of helium for injection of witness beams. Their charge and energy distribution were measured with beam position monitors and an imaging spectrometer.

\section{B. Variation of the injector profile}

An attenuator allowed adjusting the torch laser pulse energy up to $E_{\mathrm{L}} \approx 5.1 \mathrm{~mJ}$, corresponding to focus intensities up to $I_{\mathrm{L}} \approx 1.2 \times 10^{16} \mathrm{~W} / \mathrm{cm}^{2}$. Varying the laser energy and the corresponding intensity profile changed the volume and shape of the plasma torch density distribution $n_{\mathrm{T}}(x, y, z)$ according to intensity-dependent tunneling ionization rates $[53,58-$ 62]. Larger $E_{\mathrm{L}}$ produce additional $\mathrm{He}^{+}$and $\mathrm{He}^{2+}$ at higher intensities, resulting in steeper and steplike plasma density gradients.

Figure 3 shows simulations resembling the experimental configuration at FACET for three different torch laser energies
$E_{\mathrm{L}} \approx 0.5,1.0$, and $5.0 \mathrm{~mJ}$. With increasing energy, both the peak plasma density of the torch and its radial extent grow as visualized in Figs. 3(a), 3(d), and 3(g). For the minimum torch laser energy $E_{\mathrm{L}} \approx 0.5 \mathrm{~mJ}$, the central peak of the arising plasma torch shown in Fig. 3(a) results from partial ionization of $\mathrm{He}$ in the center of the laser pulse. This combination of torch width, peak density, and gradient does not suffice for trapping electrons into the plasma wave from the density downramp, as visualized by representative electron trajectories. As a side note, such subthreshold laser energy conversely allows isolation and realization of the plasma photocathode scheme via optical ionization injection when the laser pulse releases electrons directly inside the blowout [29].

As shown in Fig. 3(d), doubling the torch laser energy to $E_{\mathrm{L}} \approx 1 \mathrm{~mJ}$ increases the peak torch density and provides steeper ramps by producing a fully ionized central flat-top region of $\mathrm{He}^{+}$. This causes a more pronounced deformation of the blowout such that the trajectories of electrons originating from the torch volume overlap longer with the elongating wakefield than in the previous scenario. These particles thus gain more energy and can be captured. The trajectories also indicate the region of origin of trapped electrons, which will be investigated further in Sec. IV. Here the formed beam consists of a $Q_{1 \mathrm{~mJ}} \approx 94 \mathrm{pC}$ charge, compared to $Q_{1 \mathrm{~mJ}, \mathrm{M}} \approx 105$ $\mathrm{pC}$ predicted by the model.

Further increased torch laser energy $E_{\mathrm{L}} \approx 5 \mathrm{~mJ}$ provides intensities up to $I_{\mathrm{L}} \approx 1.2 \times 10^{16} \mathrm{~W} / \mathrm{cm}^{2}$, which also ionizes the second atomic level of helium and thus increases the peak plasma density to $n_{\mathrm{T}}+n_{\mathrm{ch}} \approx 2.6 \times 10^{23} \mathrm{~m}^{-3}$. The resulting torch distribution is much wider and provides steeper density gradients, which intensifies the deformation of the plasma blowout as shown in Fig. 3(h). This configuration injects a large amount of charge $Q_{5 \mathrm{~mJ}} \approx 498 \mathrm{pC}$, again in excellent agreement with the model prediction of $Q_{5 \mathrm{~mJ}, \mathrm{M}} \approx 495 \mathrm{pC}$. After injection, the blowout structure is significantly lengthened due to the space charge of the trapped beam and is 

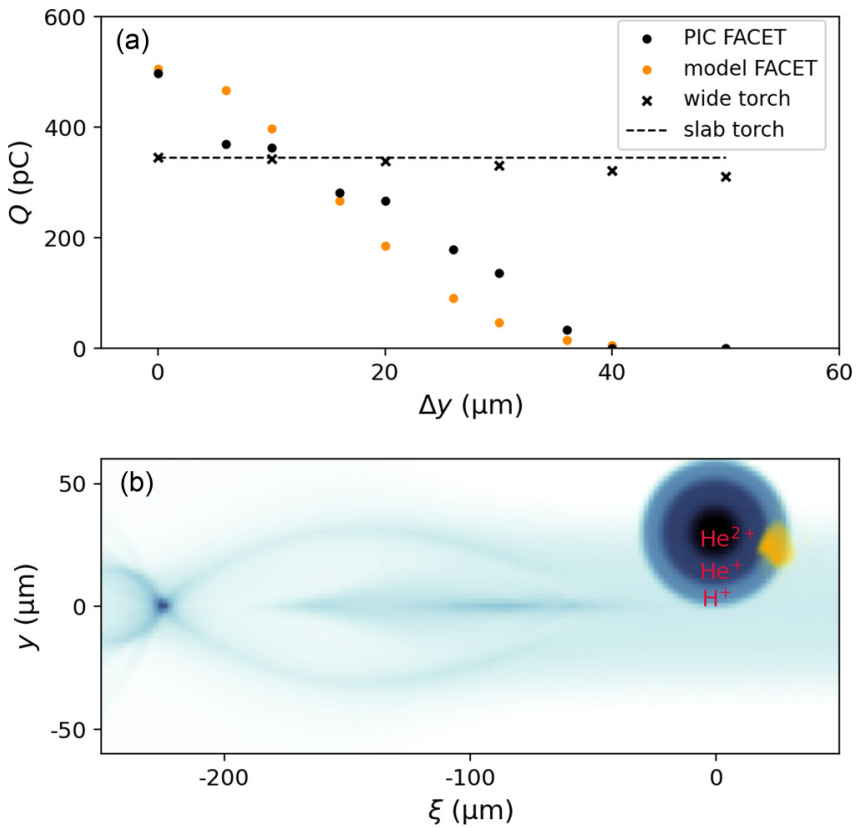

FIG. 4. Impact of transverse variation of volumetric torchwakefield overlap. (a) Injected charge as a function of transverse offset $\Delta y$ for the experimental 5-mJ case obtained from the model and PIC simulation, compared to a much wider torch with $r_{\text {core }}=$ $100 \mu \mathrm{m}$ and an infinitely wide slab. For improved stability, the two latter are implemented in a much wider channel, and for laser parameters that ionize only $\mathrm{He}^{+}$, which reduces the injected charge. (b) Simulation snapshot of the average slice electron density for $\Delta y=30 \mu \mathrm{m}$. The origin of electrons subsequently forming the witness beam is colored in yellow.

accompanied by stronger beam loading than in the previous case $[63,64]$.

These findings are consistent with experimental trends and show that witness beam charge can be tuned over hundreds of pC simply by tuning the torch laser pulse energy due to its effect on the plasma torch profile. We note that charge tunability can also be obtained from tuning of the spot size similar to Fig. 2.

\section{Variation of the injector position}

Optically generated plasma torches do not require any mechanical parts close to the interaction point and thus facilitate free positioning of the injector while maintaining undisturbed, decoupled wakefield excitation and acceleration elsewhere. This enables all-optical injection at any desired longitudinal position along the accelerator, e.g., to define the final witness beam energy. On the other hand, this also allows for transversely shifted torches that alter the volumetric overlap with the blowout. This 3D effect is unavailable with conventional $1 \mathrm{D}$ downramp injection.

Figure 4(a) shows the witness beam charges in dependence on transverse offsets $\Delta y$ based on the model and PIC simulations for the experimental scenario of laser energy $E_{\mathrm{L}} \approx 5 \mathrm{~mJ}$. Increasing the transverse torch shift reduces the overlap with the plasma wave, which decreases the injected witness charge.
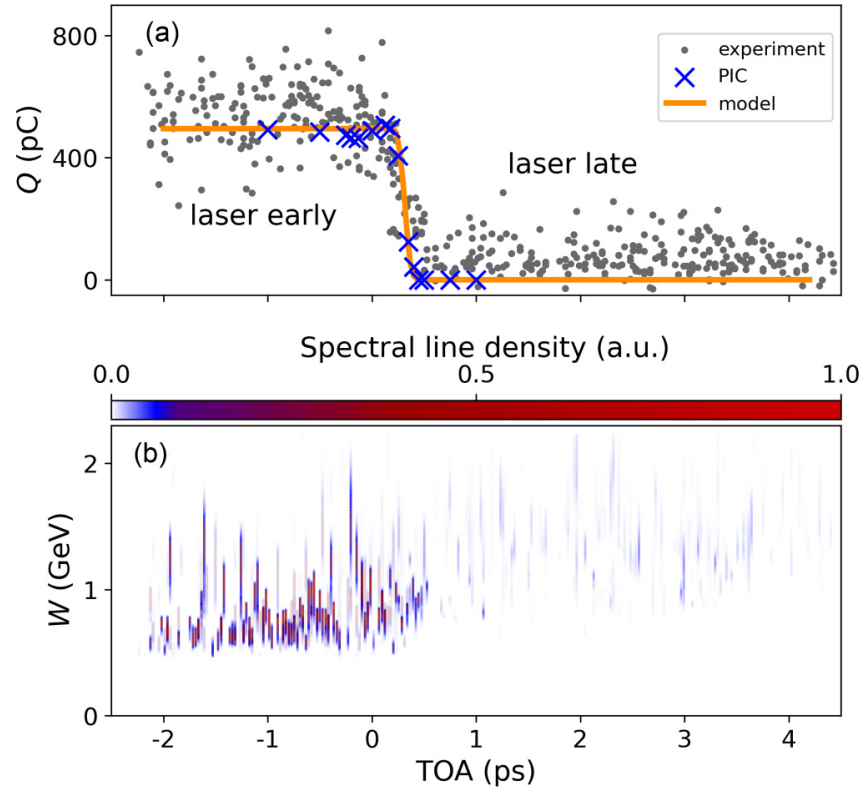

FIG. 5. Temporal variation of the torch-wave overlap. (a) Charge obtained from experiment, simulations, and model as a function of TOA. For timing TOAs less than $-0.15 \mathrm{ps}$, the torch laser arrives before the drive beam and creates a fully formed plasma torch, while for TOAs greater than $0.9 \mathrm{ps}$ the torch forms too late to interact with the wakefield. For such timings, only torch-independent dark current is measured. In the transition region, the torch is partially formed to varying degree. (b) Corresponding electron line spectra as a function of TOA.

Injection also becomes less symmetric with increasing torch offsets. For large offsets, all injected electrons originate from a dense region at one side of the blowout as shown in Fig. 4(b). Beams generated in such configurations perform large-scale centroid oscillations perpendicular to the torch axis and may be diagnosed by or exploited for betatron radiation generation.

For wider torches or slab torch geometries, the injection dynamics converge towards the established 1D longitudinal downramp picture, where the impact of transverse offsets is eliminated. It is therefore possible to realize and explore conventional 1D downramp injection and the new multidimensional downramp physics with the plasma torch approach.

\section{Variation of the injector delay}

At FACET, the relative time of arrival (TOA) between the torch-generating laser pulse and the electron drive beam was varied by an optical delay stage. The EOS setup $[65,66]$ upstream of the interaction point measured the TOA with an accuracy of $\tau_{\mathrm{EOS}} \approx 25.8 \pm 2.5 \mathrm{fs}$. This provided time stamping of the acquired data and quantified the shot-to-shot TOA jitter of $109 \pm 12 \mathrm{fs}$ (rms) obtainable at FACET.

Figure 5(a) shows an experimental TOA scan for $E_{\mathrm{L}} \approx 5$ $\mathrm{mJ}$ laser energy. For timings where the laser arrives sufficiently early, the torch is fully formed and resembles the situation simulated in Figs. 3(g)-3(i). The corresponding plateau of trapped charge of $\sim 543 \pm 97 \mathrm{pC}$ (rms) extends over 2 ps of TOA in this measurement. It could be maintained 
over hundreds of picoseconds in complementary shot series, since such cold plasma distributions evolve over nanosecond timescales $[67,68]$. Operation in the laser-early mode therefore makes injection independent of timing jitter. The charge stability of $\sim 18 \%$ observed in this demonstration of the plasma torch is already comparable to state-of-the-art (downramp-assisted) injection in LWFAs [39,69-72].

For TOA of approximately zero, the drive beam coincides with the laser pulse at the injection position, so the plasma torch is only partially formed at the arrival of the blowout. During this interaction, the laser continues to ionize and causes an overlay of plasma torch and direct ionization injection. The overlap of plasma torch and wakefield decreases for larger delays and a decreasing amount of charge is injected.

For timings where the laser pulse arrives even later, the plasma wave does not interact with the torch at all and no plasma torch injection occurs. This laser-late TOA allows quantification of dark current of $\sim 73 \pm 52 \mathrm{pC}$, which is attributed to the influence of the plasma channel on the plasma wakefield. Dark current is also present in the laserearly regime, albeit overshadowed by the much higher charge amounts injected by the plasma torch. Taking into account this dark current offset, the model from Eq. 1 and PIC simulations agree well with the experimental data.

The data show that plasma torch injection, for TOAs of approximately zero, has a temporal dependence which can be exploited. This steep transition region marks the relative TOA where electron beam and injector laser pulse overlap, and hence also represents an inherent method to quantify beamlaser synchronization. We used this aspect of torch injection to demonstrate the plasma photocathode technique in [29]. Using the transition as a marker in turn also allows one to operate the plasma torch safely in the laser-early regime, independently of shot-to-shot timing jitter.

Figure 5(b) shows the witness beam spectra measured during the TOA scan. In the laser-early regime, the beams obtain a mean energy of $\bar{W}_{\mathrm{T}} \approx 0.78 \pm 0.23 \mathrm{GeV}$ and rms energy spread of $0.05 \pm 0.03 \mathrm{GeV}$, corresponding to a relative energy spread of $6.4 \%$. Again, these values obtained in the experimental demonstration of plasma torch downramp injection are already comparable to state-of-the-art LWFA density downramp and/or ionization injectors [39,71]. Further optimization during acceleration, e.g., by beam loading and/or witness beam rephasing $[69,70,72]$, is also possible, particularly facilitated by the phase-constant nature of PWFAs.

As discussed in the following section, the injector charge and energy stability may also be further improved. Similar arguments apply to the low energy gain, which was limited by the plasma channel shape as discussed in [29].

\section{E. Optimization strategies}

We have identified major constraints and effective countermeasures to further push the performance of plasma torch downramp injectors. As discussed above, the laser-produced torch shape as well as its position and timing with respect to the wakefield determines the properties of injected beams. The obtained output-electron-beam stability is therefore strongly influenced by the shot-to-shot jitter of the incoming laser pulse. This can be mitigated by employing wide or slab torches, as shown in Fig. 4(a). However, this stabilization strategy restricts the injection capabilities to the $1 \mathrm{D}$ downramp limit. For exploitation of multidimensional injection modes, separate millijoule-level laser systems could be employed even with picosecond-level jitter when used in the laser-early regime. Such state-of-the-art laser systems offer subpercent parameter stability in terms of energy, power, pointing, and pulse quality and could be an alternative to splitoff pulses from multiple-terawatt class laser systems as used at FACET. This would substantially improve the obtainable electron-beam stability, tunability, and control. Furthermore, such low-power lasers allow kilohertz-repetition-rate operation and are therefore compatible with kilohertz-rate linear accelerators.

Another key source of shot-to-shot jitter is the plasma channel, as discussed in [29]. For example, the channel width compromised a regular plasma wave formation in our proofof-concept experiments (as seen in Fig. 3), and witness beam injection and acceleration were therefore prone to parameter jitter of the preionization laser. Furthermore, PIC simulations indicate that the observed dark current originates from the variations of the plasma channel in combination with an evolving driver beam. These experimental constraints can be straightforwardly eliminated by generating wider plasma channels, e.g., via increased preionization laser energy and/or optimized preionization optics.

The electron driver beam is the third major jitter source. Photogun-based state-of-the-art linear accelerators such as at FACET-II bear large potential for improved shot-to-shot stability and therefore nurture prospects for much higher stability from future implementations of plasma torch injectors.

Concluding, the already attractive output-beam performance observed during the experimental implementation may be significantly improved by various measures, which ensures enhanced stability and exploitation of different capabilities of plasma torch injectors.

\section{UNIQUE BEAMS FROM PLASMA TORCH INJECTION}

The above observations illustrate the general temporal and volumetric dependence of multidimensional downramp injection and the resulting tunability of witness beam charge. In the following, we show that plasma torches which are thin and steep compared to the size of the wakefield furthermore yield unique output-beam modalities. Here we use a simulation setting with strongly improved grid resolution and wide plasma channel to exclude the associated effects observed in the experiments.

Figure 6 compares injection from a wide plasma torch with injection from a narrow plasma torch by means of the respective trapping volumes $n_{\text {trap }}$, which represent the volume of origin of the plasma electrons that are captured to form the witness beams. The shape of these structures visualizes the initial conditions of the subsequently trapped electrons and in turn determines witness beam dynamics. The wide torch configuration is characterized by an annular trapping volume or "doughnut" reflecting the radial symmetry of the torch-wakefield interaction, similar to merely longitudinal gas-dynamic downramps [44,73]. The thin torch configuration, in contrast, exhibits a cropped trapping volume, 

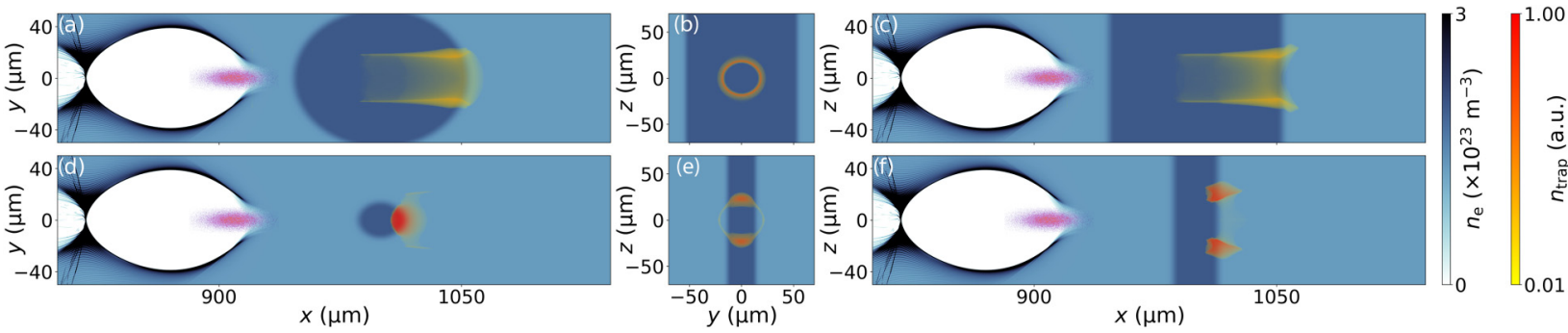

FIG. 6. Illustration of spatial torch variations on the trapping volume: (a)-(c) radially symmetric trapping volume obtained from a wide torch using $r_{\text {core }}=50 \mu \mathrm{m}$ and $l_{\text {ramp }}=10 \mu \mathrm{m}$ and (d)-(f) trapping volume from a narrow torch with $r_{\text {core }}=10 \mu \mathrm{m}$ and $l_{\text {ramp }}=10 \mu \mathrm{m}$. Here the smaller extent in $y$ reduces the volumetric overlap with the plasma wave such that injection originates predominantly from two mirrorsymmetric regions within the remaining plane in $z$.

visualizing that injection can be tailored by the volumetric overlap. In this scenario, a sufficiently thin plasma torch yields two distinct trapping volumes along the torch axis, which manifests in two mirror-symmetric electron populations that form two distinct twin beamlets.

The details of such a beamlet pair and their evolution are visualized in Fig. 7. Here each of the distinct beamlets carries $120 \mathrm{pC}$ charge and exhibits identical current, energy, and emittance distributions. Both beamlets, being born off axis, perform synchronous counteroscillating large-amplitude betatron oscillations, predominantly in the $z$ plane, and occupy distinct trace space volumes, as shown in Figs. 7(b) and 7(c), respectively. In the $y$ plane, both beamlets inhabit the same phase space area such that the total beam emittance $\epsilon_{n, y}$ equals the emittance of each beamlet. In contrast, the total beam emittance $\epsilon_{n, z}$ in the oscillation plane defined by the propagation direction of the torch laser amounts to approximately twice the emittance of each beamlet due to the mirror symmetry, as shown in Figs. 7(e) and 7(f), respectively.

Both beamlets occupy the same longitudinal phase space, as indicated in Fig. 7(d), and have low slice energy spreads. Since witness electrons in PWFAs propagate at the same velocity as the plasma wave, the injected charge can be exploited well for beam loading. This can be used to control and minimize the energy chirp the beam accumulates in the wakefield, such that the resulting spectrum becomes narrow.

Adjusting the trapping volume can be exploited not only for tuning the injected charge, but also to tune the degree
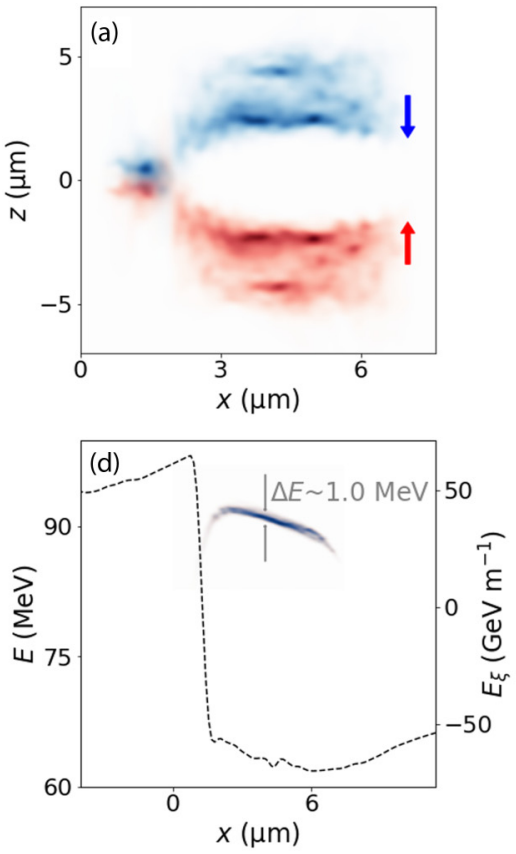
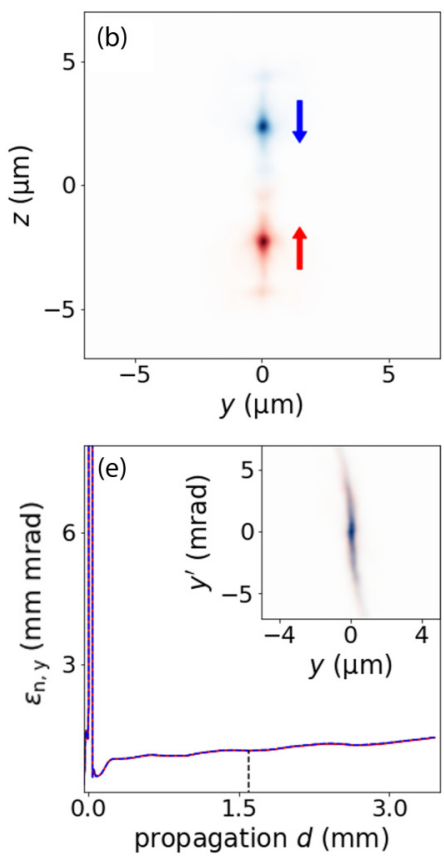
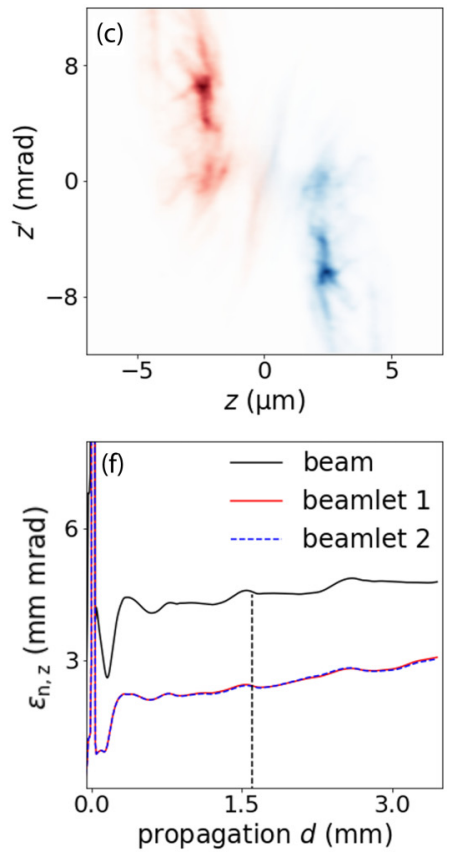

FIG. 7. Twin beamlets from reduced volumetric overlap. Shown is the witness beam from the narrow plasma torch in Fig. 6 after $\sim 1.6 \mathrm{~mm}$ of acceleration in the PWFA. Red colors correspond to the beamlet injected from the upper region where $z \geqslant 0$ and blue corresponds to injection from $z<0$. All presented particle densities are obtained from projections to the respective planes. (a) and (b) Visualization of the witness beam's planar spatial distribution, with arrows indicating the population's oscillation direction. (c) Trace space of the witness beam in the torch plane, displaying two counteroscillating populations. All panels display clear separation of the beamlets along the torch axis. The longitudinal phase space in (d) exhibits a flattened accelerating field profile from beam loading, which reduces the energy chirp. (e) and (f) Evolution of the transverse normalized emittance in both planes along the PWFA, displaying a strong asymmetry between $y$ and $z$. 


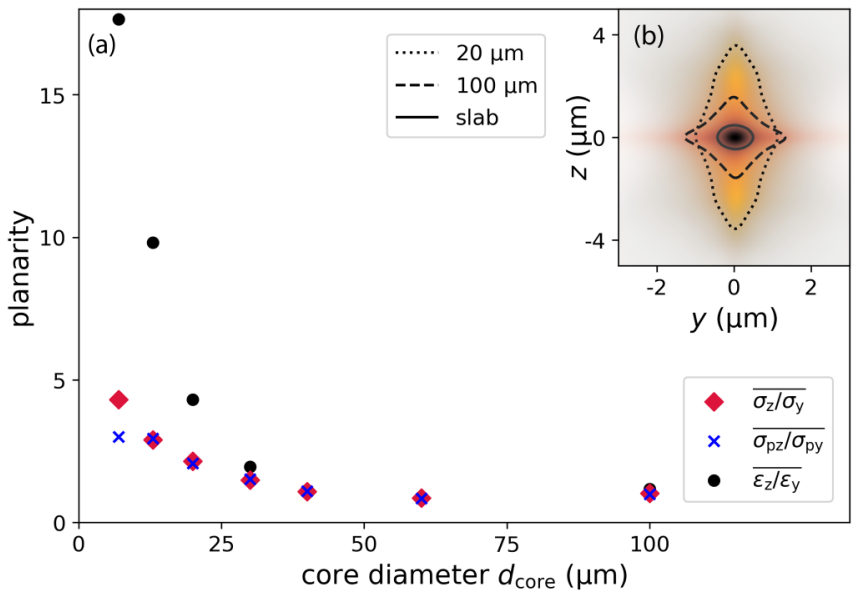

FIG. 8. Generation of planar beams from controlled volumetric overlap. (a) Aspect ratio of witness beam parameters $\sigma_{z} / \sigma_{y}, \sigma_{p z} / \sigma_{p y}$, and $\epsilon_{z} / \epsilon_{y}$ integrated over $2.5 \mathrm{~mm}$ of propagation. Beams are injected on torches with varying core diameters at constant short ramp $l_{\text {ramp }}=$ $10 \mu \mathrm{m}$. For small core diameters corresponding to reduced spatial overlap, the beams become wider in $z$ and narrow in $y$ and lead to increasingly planar distributions. (b) Projections to the transverse plane integrated over all PIC snapshots for beams from a narrow and a wide torch injector as well as the limit of an infinitely wide slab.

of planarity of the betatron oscillations. Figure 8 presents the effect of different torch core diameters on the planarity of beamlet production in terms of oscillation amplitudes, momenta, and emittances. It is evident that adequately wide plasma torches produce radially symmetric witness beams with equal transverse positions, momenta, and emittances in both planes, as expected from 1D downramp mechanics. In contrast, when reducing the torch diameter, the cropped trapping volumes inject increasingly distinct twin beamlets with increasingly planar oscillation, asymmetric emittances, and aspect ratios in both transverse planes. The degree of betatron oscillation planarity may thus be tuned by changing the plasma torch diameter, while the oscillation plane can be rotated by rotating the plasma torch laser pulse.

Planar betatron oscillation is expected to produce polarized betatron radiation, which may be exploited for light sources and imaging applications. The inherently phase-locked counteroscillation of beamlets provides another interesting feature in the context of betatron radiation sources, and exploration of its potential for diagnostics and applications is left for future work. The asymmetric emittances may also be exploited for wakefield studies [74] and collider research and development, for example, in the context of beamstrahlung [75].

The experiments at FACET have shown signatures of the potential to produce unique beam modalities by showing evidence of the effect of a temporally evolving torch during the interaction, as shown in Fig. 5, and by varying the plasma torch width via the laser energy as shown in Fig. 3, consistent with corresponding model and simulation. This consistency encourages designing experimental setups suitable to explore this further. Various advanced injector geometries are conceivable, such as plasma torches at nonperpendicular angles, crossed torch geometries or non-Gaussian torch laser pulses, and associated plasma torch injector profiles.

\section{CONCLUSION}

We have revealed the multidimensionality of density downramp injection, a highly successful scheme which has hitherto been exploited only in its 1D longitudinal limit. This generalization of density downramp injectors is unlocked by the plasma torch method, which can produce density structures below the spatiotemporal scales of the plasma accelerator. The shape and spatiotemporal overlap of the all-optically generated plasma density spikes with the wakefield determines the properties of injected electron beams. We established a model that reflects this and predicts the charge of the injected electron beam, in agreement with PIC simulations and experiments at SLAC FACET. The electron-beam quality produced in this demonstration of density downramp injection in PWFAs is already promising, and strategies have been identified to improve injection and acceleration stability in further installations and to exploit the unique capabilities arising from multidimensional downramp shaping. Simulation-based exploration of these capabilities demonstrates the possibility to form unique electron beams originating from tailored trapping volumes. For example, the constellation of plasma torches much thinner than the plasma wave can produce counteroscillating beamlet twins, and the planarity of oscillation can be adjusted all optically via the torch thickness. Such planar large-amplitude oscillations could be exploited for polarized betatron radiation, and the beam asymmetries may be useful for test beams in wakefield and collider studies. Our findings present an opportunity for unique electron-beam configurations from plasma wakefield accelerators and to study and exploit downramp injection beyond its 1D limit. In the future, experimental implementations may be realized not only at linear-accelerator-driven plasma accelerators, but also at compact hybrid LWFA-PWFA systems [76-78].

The all-optical plasma torch downramp injection approach is aligned with the overarching trend away from the use of mechanical structures with limited lifetimes, where injection, acceleration, diagnostics [66], focusing [79], and radiation production [80] may be provided by purely plasma-optical approaches.

\section{ACKNOWLEDGMENTS}

The FACET E-210 plasma wakefield acceleration experiment was built and operated with support from UCLA [U.S. Department of Energy (DOE) Contract No. DESC0009914], RadiaBeam Technologies (DOE Contract No. DE-SC0009533), and the FACET E200 team and DOE under Contract No. DE-AC02-76SF00515. B.H., P.S., A.S., A.F.H., T.H., and A.B. were supported by the European Research Council (ERC) under the European Union's Horizon 2020 research and innovation program (NeXource, ERC Grant Agreement No. 865877). The work was supported by STFC ST/S006214/1 PWFA-FEL, H2020 ERC NeXource, and EPSRC (Grant No. EP/N028694/1). J.R.C. acknowledges support from the National Science Foundation under Award No. PHY 1734281. M.D.L acknowledges support from the U.S. DOE Office of High Energy Physics under Award No. DE-SC0017906. This work used computational resources of the National Energy Research Scientific Computing Center, which is supported by DOE Contract 
No. DE-AC02-05CH11231, and of the Supercomputing Laboratory at King Abdullah University of Science \& Technology in Thuwal, Saudi Arabia.

\section{APPENDIX A: MODEL}

In the quasistatic approximation [81], the wake potential $\Psi(\xi, y, z)$ defines the accelerating field

$$
c E_{\xi}(\xi, y, z)=-\frac{\partial}{\partial \xi} \Psi(\xi, y, z),
$$

which is radially uniform in the nonlinear blowout regime when excited by radially symmetric drivers. This potential determines if single charged particles can reach the speed of light and get trapped inside the blowout. For an initially resting test particle in the presence of the passing wakefield, we can rewrite this potential $\Psi\left(\xi^{\prime}, y, z\right)=\lambda_{\mathrm{p}}^{-1}[\Psi(\xi, y, z) *$ $\delta(\xi, y, z)]\left(\xi^{\prime}\right)$ by means of the cross-correlation operator $*$ in longitudinal direction. Here $\lambda_{\mathrm{p}}^{-1}=\lambda_{\mathrm{p}}^{-1}\left(n_{\mathrm{ch}}\right)$ expresses the periodicity of the wake potential that is sustained by the plasma channel density $n_{\mathrm{ch}}$. Via the transition from a single particle to a function of the density transition $\delta(\xi, y, z) \rightarrow \eta\left[n_{\mathrm{T}}(\xi, y, z)\right]$, we define the associated potential

$$
\Psi_{\mathrm{T}}=\lambda_{\mathrm{p}}^{-1} \Psi * \eta
$$

To account for the change of the blowout volume as a function of $n_{\mathrm{T}}$, we incorporate $\eta=C\left(\Delta \lambda_{\mathrm{p}} / \lambda_{\mathrm{p}}\right)^{3}$, where $\Delta \lambda_{\mathrm{p}} / \lambda_{\mathrm{p}}=$ $1-\left(1+n_{\mathrm{T}} / n_{\mathrm{ch}}\right)^{-1 / 2}$ denotes the variation of the plasma period. We use $C \approx 565$ as a constant scaling factor throughout the paper, chosen to yield good agreement between the PIC simulation, experiment, and model.

Combining Eqs. (3) and (4) with $\eta$ connects the accelerating field and the torch density distribution such that $E_{\xi, T}=$ $\lambda_{\mathrm{p}}^{-1} E_{\xi} * \eta$. The model charge $Q$ is then obtained using Gauss's law

$$
Q\left(\xi^{\prime}\right)=-\frac{\epsilon_{0} C}{\lambda_{\mathrm{p}}} \iint E_{\xi} *\left(\frac{\Delta \lambda_{\mathrm{p}}}{\lambda_{\mathrm{p}}}\right)^{3} d y d z
$$

The minimum $Q_{M}=\left.Q\left(\xi^{\prime}\right)\right|_{\min \xi}$ corresponds to the overlap of the peak wakefields with the density gradient and approximates the witness beam charge provided by the downramp that can be sustained by the wakefield.

All presented model values are obtained by combining wakefield distributions generated in PIC simulations at high resolution with the respective torch distribution function [cf. Eq. 2].

\section{APPENDIX B: PARTICLE-IN-CELL SIMULATIONS}

Three-dimensional PIC simulations recapture the conditions in the experiment using a simulation box size of $500 \times$ $332 \times 332 \mu \mathrm{m}^{3}$ in $x, y$, and $z$ with cubic cells of $2 \mu \mathrm{m}$ size. The FACET drive beam is represented by 16 particles per cell (PPCs) and propagates through a preionized plasma channel distribution (eight PPCs) obtained from the experiment, as shown in Fig. 3(a). The simulation starts at the beginning of the plasma at $x=-0.2 \mathrm{~m}$ and self-consistently models the drive beam evolution until the injection point. There, various plasma torch distributions are implemented according to tunneling ionization caused by different laser configurations. All simulations presented in Sec. III use this simulation setting consistent with [29].

In Secs. II and IV, all simulations using $\lambda_{\mathrm{p}}=93 \mu \mathrm{m}$ were conducted at much higher resolution combined with an advanced solver avoiding numerical Cerenkov radiation [82] to capture the fine details of plasma torch injection and the subsequent acceleration including beam loading with high accuracy. The simulation box now spans over $167 \times 122 \times$ $122 \mu \mathrm{m}^{3}$ in $x, y$, and $z$ with asymmetric cells of $0.13 \times 0.24 \times$ $0.24 \mu \mathrm{m}^{3}$ corresponding to $114 k_{\mathrm{p}}$ in $x$ and $57 k_{\mathrm{p}}$ in $y$ and $z$. This allows for modeling the drive beam and the plasma torch injector by two PPCs and the preionized plasma volume by one PPC while maintaining smooth currents and high numbers of witness beam particles. The FACET-II-like [83] drive beam carries $1.56 \mathrm{nC}$ charge within rms dimensions $11.8 \times 3.9 \times 3.9 \mu \mathrm{m}^{3}$ in $x, y$, and $z$. It has $10 \mathrm{GeV}$ mean energy with $0.02 \%$ energy spread and normalized emittance of $100 \mathrm{~mm}$ mrad. Initialization of the electric self-fields happens in vacuum by a Poisson solver before the beam enters the cold homogeneous plasma via a smooth density ramp. The plasma torch injector region is then reached after $\sim 0.8 \mathrm{~mm}$ of propagation inside the plasma. There, various plasma torch distributions can be implemented according to different laser configurations. If not stated otherwise, the torch peak density is set to $n_{\mathrm{T}}=0.5 \times n_{\mathrm{ch}}$. All particles originating from this region are tracked along the subsequent acceleration.

For the $\lambda_{\mathrm{p}}=43 \mu \mathrm{m}$ case shown in Fig. 2(c), the simulation size and resolution was reduced. Here the grid cells span over $0.25 \times 0.50 \times 0.50 \mu \mathrm{m}^{3}$. Furthermore, the drive beam is reduced to $7.5 \times 3.0 \times 3.0 \mu \mathrm{m}^{3}(\mathrm{rms})$ containing $0.6 \mathrm{nC}$ charge.
[1] P. Chen, J. M. Dawson, R. W. Huff, and T. Katsouleas, Acceleration of Electrons by the Interaction of a Bunched Electron Beam with a Plasma, Phys. Rev. Lett. 54, 693 (1985).

[2] J. B. Rosenzweig, B. Breizman, T. Katsouleas, and J. J. Su, Acceleration and focusing of electrons in two-dimensional nonlinear plasma wake fields, Phys. Rev. A 44, R6189 (1991).

[3] J. Rosenzweig, Nonlinear plasma dynanics in the plasma wakefield accelerator, IEEE Trans. Plasma Sci. 15, 186 (1987).

[4] I. Blumenfeld, C. E. Clayton, F.-J. Decker, M. J. Hogan, C. Huang, R. Ischebeck, R. Iverson, C. Joshi, T. Katsouleas,
N. Kirby et al., Energy doubling of $42 \mathrm{GeV}$ electrons in a metrescale plasma wakefield accelerator, Nature (London) 445, 741 (2007).

[5] E. Kallos, T. Katsouleas, W. D. Kimura, K. Kusche, P. Muggli, I. Pavlishin, I. Pogorelsky, D. Stolyarov, and V. Yakimenko, High-Gradient Plasma-Wakefield Acceleration with Two Subpicosecond Electron Bunches, Phys. Rev. Lett. 100, 074802 (2008).

[6] M. Litos, E. Adli, W. An, C. I. Clarke, C. E. Clayton, Sébastien Corde, J. P. Delahaye, R. J. England, A. S. Fisher, J. Frederico 
et al., High-efficiency acceleration of an electron beam in a plasma wakefield accelerator, Nature (London) 515, 92 (2014).

[7] T. Tajima and J. M. Dawson, Laser Electron Accelerator, Phys. Rev. Lett. 43, 267 (1979).

[8] A. Pukhov and J. Meyer-ter-Vehn, Laser wake field acceleration: The highly non-linear broken-wave regime, Appl. Phys. B 74, 355 (2002).

[9] S. P. D. Mangles, C. D. Murphy, Z. Najmudin, A. G. R. Thomas, J. L. Collier, A. E. Dangor, E. J. Divall, P. S. Foster, J. G. Gallacher, C. J. Hooker et al., Monoenergetic beams of relativistic electrons from intense laser-plasma interactions, Nature (London) 431, 535 (2004).

[10] J. Faure, Y. Glinec, A. Pukhov, S. Kiselev, S. Gordienko, E. Lefebvre, J. P. Rousseau, F. Burgy, and V. Malka, A laserplasma accelerator producing monoenergetic electron beams, Nature (London) 431, 541 (2004).

[11] C. G. R. Geddes, C. S. Toth, J. Van Tilborg, E. Esarey, C. B. Schroeder, D. Bruhwiler, C. Nieter, J. Cary, and W. P. Leemans, High-quality electron beams from a laser wakefield accelerator using plasma-channel guiding, Nature (London) 431, 538 (2004).

[12] J. Faure, C. Rechatin, A. Norlin, A. Lifschitz, Y. Glinec, and V. Malka, Controlled injection and acceleration of electrons in plasma wakefields by colliding laser pulses, Nature (London) 444, 737 (2006).

[13] J. M. Dawson, Nonlinear electron oscillations in a cold plasma, Phys. Rev. 113, 383 (1959).

[14] A. I. Akhiezer and R. V. Polovin, Theory of wave motion of an electron plasma, Sov. Phys. JETP 3, 696 (1956).

[15] W. K. Panofsky, Evolution of particle accelerators, SLAC Beam Line 27, 36 (1997).

[16] T. Tajima and V. Malka, Laser plasma accelerators, Plasma Phys. Contr. Fusion 62, 034004 (2020).

[17] S. Bulanov, N. Naumova, F. Pegoraro, and J. Sakai, Particle injection into the wave acceleration phase due to nonlinear wake wave breaking, Phys. Rev. E 58, R5257 (1998).

[18] H. Suk, N. Barov, J. B. Rosenzweig, and E. Esarey, in The Physics OfHigh Brightness Beams, edited by J. Rosenzweig and L. Serafini (World Scientific, Singapore, 2000), pp. 404-417.

[19] D. Umstadter, J. K. Kim, and E. Dodd, Laser Injection of Ultrashort Electron Pulses into Wakefield Plasma Waves, Phys. Rev. Lett. 76, 2073 (1996).

[20] E. Esarey, R. F. Hubbard, W. P. Leemans, A. Ting, and P. Sprangle, Electron Injection into Plasma Wakefields by Colliding Laser Pulses, Phys. Rev. Lett. 79, 2682 (1997).

[21] J. U. Kim, N. Hafz, and H. Suk, Electron trapping and acceleration across a parabolic plasma density profile, Phys. Rev. E 69 , 026409 (2004).

[22] G. Fubiani, E. Esarey, C. B. Schroeder, and W. P. Leemans, Improvement of electron beam quality in optical injection schemes using negative plasma density gradients, Phys. Rev. E 73, 026402 (2006).

[23] M. Chen, Z. M. Sheng, Y. Y. Ma, and J. Zhang, Electron injection and trapping in a laser wakefield by field ionization to high-charge states of gases, J. Appl. Phys. 99, 056109 (2006).

[24] B. Hidding, G. Pretzler, J. B. Rosenzweig, T. Königstein, D. Schiller, and D. L. Bruhwiler, Ultracold Electron Bunch Generation via Plasma Photocathode Emission and Acceleration in a Beam-Driven Plasma Blowout, Phys. Rev. Lett. 108, 035001 (2012).
[25] T. Y. Chien, C. L. Chang, C. H. Lee, J. Y. Lin, J. Wang, and S. Y. Chen, Spatially Localized Self-Injection of Electrons in a Self-Modulated Laser-Wakefield Accelerator by Using a LaserInduced Transient Density Ramp, Phys. Rev. Lett. 94, 115003 (2005).

[26] E. Oz, S. Deng, T. Katsouleas, P. Muggli, C. D. Barnes, I. Blumenfeld, F. J. Decker, P. Emma, M. J. Hogan, R. Ischebeck et al., Ionization-Induced Electron Trapping in Ultrarelativistic Plasma Wakes, Phys. Rev. Lett. 98, 084801 (2007).

[27] C. G. R. Geddes, K. Nakamura, G. R. Plateau, C. Toth, E. Cormier-Michel, E. Esarey, C. B. Schroeder, J. R. Cary, and W. P. Leemans, Plasma-Density-Gradient Injection of Low Absolute-Momentum-Spread Electron Bunches, Phys. Rev. Lett. 100, 215004 (2008).

[28] N. Vafaei-Najafabadi, K. A. Marsh, C. E. Clayton, W. An, W. B. Mori, C. Joshi, W. Lu, E. Adli, S. Corde, M. Litos et al., Beam Loading by Distributed Injection of Electrons in a Plasma Wakefield Accelerator, Phys. Rev. Lett. 112, 025001 (2014).

[29] A. Deng, O. S. Karger, T. Heinemann, A. Knetsch, P. Scherkl, G. G. Manahan, A. Beaton, D. Ullmann, G. Wittig, A. F. Habib et al., Generation and acceleration of electron bunches from a plasma photocathode, Nat. Phys. 15, 1156 (2019).

[30] J. Faure, C. Rechatin, O. Lundh, L. Ammoura, and V. Malka, Injection and acceleration of quasimonoenergetic relativistic electron beams using density gradients at the edges of a plasma channel, Phys. Plasmas 17, 083107 (2010).

[31] P. Brijesh, C. Thaury, K. T. Phuoc, S. Corde, G. Lambert, V. Malka, S. P. D. Mangles, M. Bloom, and S. Kneip, Tuning the electron energy by controlling the density perturbation position in laser plasma accelerators, Phys. Plasmas 19, 063104 (2012).

[32] A. J. Gonsalves, K. Nakamura, C. Lin, D. Panasenko, S. Shiraishi, T. Sokollik, C. Benedetti, C. B. Schroeder, C. G. R. Geddes, J. Van Tilborg et al., Tunable laser plasma accelerator based on longitudinal density tailoring, Nat. Phys. 7, 862 (2011).

[33] M. Hansson, B. Aurand, X. Davoine, H. Ekerfelt, K. Svensson, A. Persson, C. G. Wahlström, and O. Lundh, Down-ramp injection and independently controlled acceleration of electrons in a tailored laser wakefield accelerator, Phys. Rev. ST Accel. Beams 18, 071303 (2015).

[34] K. Schmid, A. Buck, C. M. S. Sears, J. M. Mikhailova, R. Tautz, D. Herrmann, M. Geissler, F. Krausz, and L. Veisz, Densitytransition based electron injector for laser driven wakefield accelerators, Phys. Rev. ST Accel. Beams 13, 091301 (2010).

[35] A. Buck, J. Wenz, J. Xu, K. Khrennikov, K. Schmid, M. Heigoldt, J. M. Mikhailova, M. Geissler, B. Shen, F. Krausz et al., Shock-Front Injector for High-Quality Laser-Plasma Acceleration, Phys. Rev. Lett. 110, 185006 (2013).

[36] S. K. Barber, J. van Tilborg, C. B. Schroeder, R. Lehe, H. E. Tsai, K. K. Swanson, S. Steinke, K. Nakamura, C. G. R. Geddes, C. Benedetti et al., Measured Emittance Dependence on the Injection Method in Laser Plasma Accelerators, Phys. Rev. Lett. 119, 104801 (2017).

[37] K. K. Swanson, H.-E. Tsai, S. K. Barber, R. Lehe, H.-S. Mao, S. Steinke, J. van Tilborg, K. Nakamura, C. G. R. Geddes, C. B. Schroeder et al., Control of tunable, monoenergetic laser-plasma-accelerated electron beams using a shock-induced density downramp injector, Phys. Rev. Accel. Beams 20, 051301 (2017). 
[38] M. Burza, A. Gonoskov, K. Svensson, F. Wojda, A. Persson, M. Hansson, G. Genoud, M. Marklund, C. G. Wahlström, and O. Lundh, Laser wakefield acceleration using wire produced double density ramps, Phys. Rev. ST Accel. Beams 16, 011301 (2013).

[39] J. Wenz, A. Döpp, K. Khrennikov, S. Schindler, M. F. Gilljohann, H. Ding, J. Götzfried, A. Buck, J. Xu, M. Heigoldt, W. Helml, L. Veisz, and S. Karsch, Dual-energy electron beams from a compact laser-driven accelerator, Nat. Photon. 13, 263 (2019).

[40] X. L. Xu, F. Li, W. An, T. N. Dalichaouch, P. Yu, W. Lu, C. Joshi, and W. B. Mori, High quality electron bunch generation using a longitudinal density-tailored plasma-based accelerator in the three-dimensional blowout regime, Phys. Rev. Accel. Beams 20, 111303 (2017).

[41] C. Joshi, E. Adli, W. An, C. E. Clayton, S. Corde, S. Gessner, M. J. Hogan, M. Litos, W. Lu, K. A. Marsh et al., Plasma wakefield acceleration experiments at FACET II, Plasma Phys. Contr. Fusion 60, 034001 (2018).

[42] C. Zhang, C. K. Huang, K. A. Marsh, X. L. Xu, F. Li, M. Hogan, V. Yakimenko, S. Corde, W. B. Mori, and C. Joshi, Effect of fluctuations in the down ramp plasma source profile on the emittance and current profile of the self-injected beam in a plasma wakefield accelerator, Phys. Rev. Accel. Beams 22, 111301 (2019).

[43] J. Grebenyuk, A. M. de la Ossa, T. Mehrling, and J. Osterhoff, Beam-driven plasma-based acceleration of electrons with density down-ramp injection at flashforward, Nucl. Instrum. Methods Phys. Res. A 740, 246 (2014).

[44] A. Martinez de la Ossa, Z. Hu, M. J. V. Streeter, T. J. Mehrling, O. Kononenko, B. Sheeran, and J. Osterhoff, Optimizing density down-ramp injection for beam-driven plasma wakefield accelerators, Phys. Rev. Accel. Beams 20, 091301 (2017).

[45] R. J. England, J. B. Rosenzweig, and N. Barov, Plasma electron fluid motion and wave breaking near a density transition, Phys. Rev. E 66, 016501 (2002).

[46] M. C. Thompson, J. B. Rosenzweig, and H. Suk, Plasma density transition trapping as a possible high-brightness electron beam source, Phys. Rev. ST Accel. Beams 7, 011301 (2004).

[47] A. Aschikhin, C. Behrens, S. Bohlen, J. Dale, N. Delbos, L. di Lucchio, E. Elsen, J.-H. Erbe, M. Felber, B. Foster, L. Goldberg, J. Grebenyuk, J.-N. Gruse, B. Hidding, Zhanghu Hu, S. Karstensen, A. Knetsch, O. Kononenko, V. Libov, K. Ludwig et al., The FLASHForward facility at DESY, Nucl. Instrum. Methods Phys. Res. A 806, 175 (2016).

[48] G. Wittig, O. Karger, A. Knetsch, Y. Xi, A. Deng, J. B. Rosenzweig, D. L. Bruhwiler, J. Smith, G. G. Manahan, Z. M. Sheng et al., Optical plasma torch electron bunch generation in plasma wakefield accelerators, Phys. Rev. ST Accel. Beams 18, 081304 (2015).

[49] G. Wittig, O. S. Karger, A. Knetsch, Y. Xi, A. Deng, J. B. Rosenzweig, D. L. Bruhwiler, J. Smith, Z. M. Sheng, D. A. Jaroszynski et al., Electron beam manipulation, injection and acceleration in plasma wakefield accelerators by optically generated plasma density spikes, Nucl. Instrum. Methods Phys. Res. A 829, 83 (2016).

[50] See Supplemental Material at http://link.aps.org/supplemental/ 10.1103/PhysRevResearch.3.043163 for a video representation of a plasma torch injector shown in Fig. 1.
[51] C. Nieter and J. R. Cary, Vorpal: A versatile plasma simulation code, J. Comput. Phys. 196, 448 (2004).

[52] H. Ekerfelt, M. Hansson, I. G. González, X. Davoine, and O. Lundh, A tunable electron beam source using trapping of electrons in a density down-ramp in laser wakefield acceleration, Sci. Rep. 7, 12229 (2017).

[53] D. L. Bruhwiler, D. A. Dimitrov, J. R. Cary, E. Esarey, W. Leemans, and R. E. Giacone, Particle-in-cell simulations of tunneling ionization effects in plasma-based accelerators, Phys. Plasmas 10, 2022 (2003).

[54] H.-y. Suk, G.-H. Kim, J.-U. Kim, C.-B. Kim, and H.-J. Lee, Method of trapping accelerating electrons in plasma, U.S. Patent No. US 7,049,736 B2 (23 May 2006).

[55] G. G. Manahan, A. F. Habib, P. Scherkl, P. Delinikolas, A. Beaton, A. Knetsch, O. Karger, G. Wittig, T. Heinemann, Z. M. Sheng et al., Single-stage plasma-based correlated energy spread compensation for ultrahigh 6D brightness electron beams, Nat. Commun. 8, 15705 (2017).

[56] N. Davidson, A. A. Friesem, and E. Hasman, Holographic axilens: High resolution and long focal depth, Opt. Lett. 16, 523 (1991).

[57] S. Z. Green, E. Adli, C. I. Clarke, S. Corde, S. A. Edstrom, A. S. Fisher, J. Frederico, J. C. Frisch, S. Gessner, S. Gilevich et al., Laser ionized preformed plasma at facet, Plasma Phys. Contr. Fusion 56, 084011 (2014).

[58] A. I. Nikishov and V. I. Ritus, Ionization of atoms by an electromagnetic-wave field, Sov. Phys. JETP 25, 145 (1967).

[59] A. M. Perelomov and V. S. Popov, Ionization of atoms in an alternating electrical field. III, Sov. Phys. JETP 25, 336 (1967).

[60] A. M. Perelomov, V. S. Popov, and M. V. Terentev, Ionization of atoms in an alternating electric field, Sov. Phys. JETP 23, 924 (1966).

[61] A. I. Nikishov and V. I. Ritus, Ionization of systems bound by short-range forces by the field of an electromagnetic wave, Sov. Phys. JETP 23, 168 (1966).

[62] M. V. Ammosov, N. B. Delone, and V. P. Krainov, Tunnel ionization of complex atoms and atomic ions in a varying electromagnetic-field, Sov. Phys. JETP 91, 2008 (1986).

[63] S. Wilks, T. Katsouleas, J. M. Dawson, P. Chen, and J. J. Su, Beam loading in plasma waves, IEEE Trans. Plasma Sci. 15, 210 (1987).

[64] M. Tzoufras, W. Lu, F. S. Tsung, C. Huang, W. B. Mori, T. Katsouleas, J. Vieira, R. A. Fonseca, and L. O. Silva, Beam Loading in the Nonlinear Regime of Plasma-Based Acceleration, Phys. Rev. Lett. 101, 145002 (2008).

[65] X. Yan, A. M. MacLeod, W. A. Gillespie, G. M. H. Knippels, D. Oepts, A. F. G. van der Meer, and W. Seidel, Subpicosecond Electro-Optic Measurement of Relativistic Electron Pulses, Phys. Rev. Lett. 85, 3404 (2000).

[66] P. Scherkl, A. Knetsch, T. Heinemann, A. Sutherland, A. F. Habib, O. Karger, D. Ullmann, A. Beaton, G. Kirwan, G. Manahan et al., Plasma-photonic spatiotemporal synchronization of relativistic electron and laser beams, arXiv:1908.09263.

[67] C. G. Durfee and H. M. Milchberg, Light Pipe for High Intensity Laser Pulses, Phys. Rev. Lett. 71, 2409 (1993).

[68] R. J. Shalloo, C. Arran, L. Corner, J. Holloway, J. Jonnerby, R. Walczak, H. M. Milchberg, and S. M. Hooker, Hydrodynamic optical-field-ionized plasma channels, Phys. Rev. E 97, 053203 (2018). 
[69] W. T. Wang, W. T. Li, J. S. Liu, Z. J. Zhang, R. Qi, C. H. Yu, J. Q. Liu, M. Fang, Z. Y. Qin, C. Wang, Y. Xu, F. X. Wu, Y. X. Leng, R. X. Li, and Z. Z. Xu, High-Brightness High-Energy Electron Beams from a Laser Wakefield Accelerator Via Energy Chirp Control, Phys. Rev. Lett. 117, 124801 (2016).

[70] W. Wang, K. Feng, L. Ke, C. Yu, Y. Xu, R. Qi, Y. Chen, Z. Qin, Z. Zhang, M. Fang et al., Free-electron lasing at 27 nanometres based on a laser wakefield accelerator, Nature (London) $\mathbf{5 9 5}$, $516(2021)$.

[71] A. R. Maier, N. M. Delbos, T. Eichner, L. Hübner, S. Jalas, L. Jeppe, S. W. Jolly, M. Kirchen, V. Leroux, P. Messner, M. Schnepp, M. Trunk, P. A. Walker, C. Werle, and P. Winkler, Decoding Sources of Energy Variability in a Laser-Plasma Accelerator, Phys. Rev. X 10, 031039 (2020).

[72] M. Kirchen, S. Jalas, P. Messner, P. Winkler, T. Eichner, L. Hübner, T. Hülsenbusch, L. Jeppe, T. Parikh, M. Schnepp, and A. R. Maier, Optimal Beam Loading in a Laser-Plasma Accelerator, Phys. Rev. Lett. 126, 174801 (2021).

[73] A. Döpp, B. Mahieu, A. Lifschitz, C. Thaury, A. Doche, E. Guillaume, G. Grittani, O. Lundh, M. Hansson, J. Gautier et al., Stable femtosecond $\mathrm{x}$-rays with tunable polarization from a laser-driven accelerator, Light: Sci. Appl. 6, e17086 (2017).

[74] S. S. Baturin, G. Andonian, and J. B. Rosenzweig, Analytical treatment of the wakefields driven by transversely shaped beams in a planar slow-wave structure, Phys. Rev. Accel. Beams 21, 121302 (2018).

[75] P. Chen and V. I. Telnov, Coherent Pair Creation in Linear Colliders, Phys. Rev. Lett. 63, 1796 (1989).

[76] B. Hidding, T. Koenigstein, J. Osterholz, S. Karsch, O. Willi, and G. Pretzler, Monoenergetic Energy Doubling in a
Hybrid Laser-Plasma Wakefield Accelerator, Phys. Rev. Lett. 104, 195002 (2010).

[77] M. F. Gilljohann, H. Ding, A. Doepp, J. Goetzfried, S. Schindler, G. Schilling, S. Corde, A. Debus, T. Heinemann, B. Hidding et al., Direct Observation of Plasma Waves and Dynamics Induced by Laser-Accelerated Electron Beams, Phys. Rev. X 9, 011046 (2019).

[78] T. Kurz, T. Heinemann, M. F. Gilljohann, Y. Y. Chang, J. P. Couperus Cabadağ, A. Debus, O. Kononenko, R. Pausch, S. Schöbel, R. W. Assmann, M. Bussmann, H. Ding, J. Götzfried, A. Köhler, G. Raj, S. Schindler, K. Steiniger, O. Zarini, S. Corde, A. Döpp et al., Demonstration of a compact plasma accelerator powered by laser-accelerated electron beams, Nat. Commun. 12, 2895 (2021).

[79] C. E. Doss, E. Adli, R. Ariniello, J. Cary, S. Corde, B. Hidding, M. J. Hogan, K. Hunt-Stone, C. Joshi, K. A. Marsh et al., Laser-ionized, beam-driven, underdense, passive thin plasma lens, Phys. Rev. Accel. Beams 22, 111001 (2019).

[80] I. A. Andriyash, R. Lehe, A. Lifschitz, C. Thaury, J.-M. Rax, K. Krushelnick, and V. Malka, An ultracompact x-ray source based on a laser-plasma undulator, Nat. Commun. 5, 4736 (2014).

[81] P. Sprangle, E. Esarey, and A. Ting, Nonlinear Theory of Intense Laser-Plasma Interactions, Phys. Rev. Lett. 64, 2011 (1990).

[82] B. M. Cowan, D. L. Bruhwiler, J. R. Cary, E. Cormier-Michel, and C. G. R. Geddes, Generalized algorithm for control of numerical dispersion in explicit time-domain electromagnetic simulations, Phys. Rev. ST Accel. Beams 16, 041303 (2013).

[83] V. Yakimenko, L. Alsberg, E. Bong, G. Bouchard, C. Clarke, C. Emma, S. Green, C. Hast, M. J. Hogan, J. Seabury et al., FACET-II facility for advanced accelerator experimental tests, Phys. Rev. Accel. Beams 22, 101301 (2019). 